\title{
MIXED PROBLEM WITH BOUNDARY INTEGRAL CONDITIONS FOR A CERTAIN PARABOLIC EQUATION
}

\author{
ABDELFATAH BOUZIANI \\ Institut National d'Enseignement \\ Supérieur de Mécanique \\ Oum El Bouaghi, Algérie
}

(Received October, 1994; Revised December, 1995)

\begin{abstract}
The present article is devoted to a proof of the existence and uniqueness of a solution of a mixed problem with boundary integral conditions for a certain parabolic equation. The proof is based on an energy inequality and on the fact that the range of the operator generated by the problem is dense.
\end{abstract}

Key words: Parabolic Equation, Boundary Integral Conditions, Energy Inequality.

AMS (MOS) subject classifications: $34 \mathrm{~B} 99,34 \mathrm{~B} 15$.

\section{Introduction}

In the rectangle $Q=(0, b) \times(0, T)$, we consider the equation

$$
\mathcal{L} u=\frac{\partial u}{\partial t}+(-1)^{m} a(t) \frac{\partial^{2 m} u}{\partial x^{2 m}}=f(x, t),
$$

where $a(t)$ is bounded, $0<a_{0} \leq a(t) \leq a_{1}$, and $a(t)$ has the bounded derivative such that $0<c_{0} \leq$ $a^{\prime}(t) \leq c_{1}$ for $t \in[0, T]$.

We adhere to equation (1.1) the initial condition

$$
\ell u=u(x, 0)=\varphi(x)
$$

and the boundary conditions

$$
\int_{0}^{b} x^{k} \cdot u(x, t) d x=0, \quad k=\overline{0,2 m-1} .
$$

The importance of problems with integral conditions has been pointed out by Samarskii [9]. Problems which combine local and integral condition for second order parabolic equations are investigated by the potential method [2, 7], by Fourier's method [4-6], and by the energy inequalities method $[1,8,10]$.

In this paper, the existence and uniqueness of a solution of problem (1.1)-(1.3) is proved. The proof is based on the method of energy inequalities, presented in [1]. Such problems have not been studied previously. 


\section{Preliminaries}

First, we introduce the appropriate function spaces which will be used in the paper. We denote $B_{2}^{m}(0, b)$ by:

$$
B_{2}^{m}(0, b):=\left\{\begin{array}{cc}
L^{2}(0, b) & \text { for } m=0 \\
\left\{u / \sigma^{m} u \in L^{2}(0, b)\right\} & \text { for } m \geq 1
\end{array}\right.
$$
where $\mathcal{G}^{m} u:=\int_{0}^{x} \frac{(x-\xi)^{m-1}}{(m-1) !} u(\xi, t) d \xi, m \geq 1$. For $m \geq 1$, the scalar product in $B_{2}^{m}(0, b)$ is de-
fined by:

The associated norm is:

$$
(u, v)_{B_{2}^{m}(0, b)}=\int_{0}^{b} \mathcal{T}^{m} u \mathcal{T}^{m} v d x .
$$

$$
\|u\|_{B_{2}^{m}(0, b)}=\left\|\sigma^{m} u\right\|_{L^{2}(0, b)} \text { for } m \geq 1
$$

Lemma 1: For $m \in \mathbb{N}$, we have

$$
\|u\|_{B_{2}^{m}(0, b)}^{2} \leq \frac{b^{2}}{2}\|u\|_{B_{2}^{m-1}(0, b)}^{2} .
$$

Proof: The Cauchy-Schwarz inequality gives

$$
\begin{aligned}
\left|\mathcal{\sigma}^{m} u\right|^{2} \leq & \left|\int_{0}^{x} \mathcal{\sigma}^{m-1} u(\xi, t) d \xi\right|^{2} \leq\left(\int_{0}^{x} d \xi\right) \cdot\left(\int_{0}^{x}\left|\mathcal{\sigma}^{m-1} u(\xi, t)\right|^{2} d \xi\right) \\
& \leq x \cdot \int_{0}^{x}\left|\mathcal{\sigma}^{m-1} u(\xi, t)\right|^{2} d \xi \leq x \cdot \int_{0}^{b}\left|\mathcal{T}^{m-1} u(\xi, t)\right|^{2} d \xi .
\end{aligned}
$$

Therefore, we have

$$
\begin{aligned}
\|u\|_{B_{2}^{m}(0, b)}^{2} & \leq \int_{0}^{b}\left|\sigma^{m-1} u(\xi, t)\right|^{2} d \xi \cdot \int_{0}^{b} x d x \\
& =\frac{b^{2}}{2}\|u\|_{B_{2}^{m-1}(0, b)}^{2}
\end{aligned}
$$

Corollary: For $m \in \mathbb{N}$, we have

$$
\|u\|_{B_{2}^{m}(0, b)}^{2} \leq\left(\frac{b^{2}}{2}\right)^{m} \cdot\|u\|_{L^{2}(0, b)}^{2} .
$$

Remark: Inequalities (2.2) and (2.3) remain valid, if we replace the interval $(0, b)$ by a bounded region $\Omega$ of $\mathbb{R}^{n}$. It suffices to replace $b$ by meas $(\Omega)$ (measure of $\Omega$ ) in (2.2) and (2.3).

The space $B_{2}^{m, k}(Q)$ is the space with the finite norm

$$
\|u\|_{B_{2}^{m, k}(Q)}^{2}=\int_{0}^{T}\|u(\cdot, t)\|_{B_{2}^{m}(0, b)}^{2} d t+\int_{0}^{b}\|u(x, \cdot)\|_{B_{2}^{k}(0, T)}^{2} d x
$$


The space $B_{2}^{0,0}(Q)$ coincides with $L^{2}(Q)$.

We associate with problem (1.1)-(1.3), the operator $L=(\ell, \ell)$ with domain denoted by $D(L)=: E$. The operator $L$ is from $E$ to $F ; E$ is Banach space of the functions $u \in L^{2}(0, b)$ satisfying (1.3), with the finite norm

$$
\|u\|_{E}^{2}=\left\|\frac{\partial u}{\partial t}\right\|_{B_{2}^{m, 0}(Q)}^{2}+\left\|\frac{\partial^{2 m} u}{\partial x^{2 m}}\right\|_{B_{2}^{m, 0}(Q)_{0} \leq \tau \leq T}^{+\sup _{\leq}}\|u(x, \tau)\|_{L^{2}(0, b)}^{2},
$$

where $F$ is the Hilbert space obtained by completing the space $B_{2}^{m, 0}(Q) \times L^{2}(0, b)$ equipped with the norm

$$
\|\mathscr{F}\|_{F}^{2}=\|f\|_{B_{2}^{m, 0}(Q)}^{2}+\|\varphi\|_{L^{2}(0, b)}^{2}, \mathscr{F}=(f, \varphi) .
$$

Here, we assumed that the function $\varphi$ satisfies the conditions in the form (1.3), i.e.,

$$
\int_{0}^{b} x^{k} \cdot \varphi d x=0, \quad k=\overline{0,2 m-1}
$$

\section{Two-Sided A Priori Estimates}

Theorem 1: The following a priori estimate

$$
\|L u\|_{F} \leq c\|u\|_{E}
$$

holds for any function $u \in E$, where constant $c$ is independent of $u$.

Proof: Equation (1.1) implies that

$$
\|\ell u\|_{B_{2}^{m, 0}(Q)}^{2} \leq 2\left(\left\|\frac{\partial u}{\partial t}\right\|_{B_{2}^{m, 0}(Q)}^{2}+a_{1}^{2}\left\|\frac{\partial^{2 m} u}{\partial x^{2 m}}\right\|_{B_{2}^{m, 0}(Q)}^{2}\right)
$$

and initial condition (1.2) yields

$$
\|\mathcal{L} u\|_{L^{2}(0, b)}^{2} \leq \sup _{0 \leq \tau \leq T}\|u(x, \tau)\|_{L^{2}(0, b)}^{2}
$$

Combining inequality (3.2) with (3.3), we obtain (3.1) for $u \in E$, with $c$ : $=\max \left(2^{1 / 2}, 2^{1 / 2} a_{1}\right)$.

Theorem 2: For any function $u \in E$, we have the inequality

$$
\|u\|_{E} \leq c\|L u\|_{F}
$$

where constant $c>0$ does not depend on $u$.

Proof: We consider the scalar product in $L^{2}\left(Q^{\tau}\right)$, where $Q^{\tau}:=(0, b) \times(0, \tau)$ and $0 \leq \tau \leq T$. Observe that

$$
\begin{aligned}
& 2 \int_{Q^{\tau}}\left|\sigma^{m} \frac{\partial u}{\partial t}\right|^{2} d x d t+\int_{0}^{b} a(\tau)|u(x, \tau)|^{2} d x \\
& =2 \operatorname{Re}\left(\ell u,(-1)^{m_{\mathcal{J}^{2}}} \frac{m}{\partial \bar{u}} \frac{{ }^{\prime}}{\partial t}\right)_{0} \tau+\int_{0}^{b} a(0)|\varphi|^{2} d x+\int_{Q^{\tau}} a^{\prime}(t)|u|^{2} d x d t
\end{aligned}
$$


We estimate the first term on the right-hand side of (3.5). By applying an elementary inequality we have

$$
2 \operatorname{Re}\left(\mathcal{L} u,(-1)^{m \mathcal{J}^{2 m}} \frac{\partial \bar{u}}{\partial t}\right)_{0, Q^{\tau}} \leq\|\mathcal{L} u\|_{B_{2}^{m, 0}\left(Q^{\tau}\right)}^{2}+\left\|\frac{\partial u}{\partial t}\right\|_{B_{2}^{m, 0}\left(Q^{\tau}\right)}^{2}
$$

From equation (1.1), we obtain

$$
\frac{1}{4} a_{0}^{2}\left\|\frac{\partial^{2 m} u}{\partial x^{2 m}}\right\|_{B_{2}^{m, 0}\left(Q^{\tau}\right)}^{2} \leq \frac{1}{2}\left\|\frac{\partial u}{\partial t}\right\|_{B_{2}^{m, 0}\left(Q^{\tau}\right)}^{2}+\frac{1}{2}\|\mathcal{L} u\|_{B_{2}^{m, 0}\left(Q^{\tau}\right)}^{2}
$$

Therefore, by formulas (3.5)-(3.7),

$$
\begin{gathered}
\frac{1}{2}\left\|\frac{\partial u}{\partial t}\right\|_{B_{2}^{m, 0}\left(Q^{\tau}\right)}^{2}+\frac{1}{4} a_{0}^{2}\left\|\frac{\partial^{2 m} u}{\partial x^{2 m}}\right\|_{B_{2}^{m, 0}\left(Q^{\tau}\right)}^{2}+a_{0}\|u(x, \tau)\|_{L^{2}(0, b)}^{2} \\
\leq \frac{3}{2}\|\ell u\|_{B_{2}^{m, 0}\left(Q^{\tau}\right)}^{2}+a_{1}\|\ell u\|_{L^{2}(0, b)}^{2}+c_{1}\|u\|_{L^{2}\left(Q^{\tau}\right)^{\tau}}^{2}
\end{gathered}
$$

Applying Lemma 7.1 from [3] to the above inequality we get

where

$$
\begin{gathered}
\left\|\frac{\partial u}{\partial t}\right\|_{B_{2}^{m, 0}\left(Q^{\tau}\right)}^{2}+\left\|\frac{\partial^{2 m} u}{\partial x^{2 m}}\right\|_{B_{2}^{m, 0}\left(Q^{\tau}\right)}^{2}+\|u(x, \tau)\|_{L^{2}(0, b)}^{2} \\
\leq c_{2}\left(\|\mathcal{L} u\|_{B_{2}^{m, 0}(Q)}^{2}+\|\ell u\|_{L^{2}(0, b)}^{2}\right)
\end{gathered}
$$

$$
c_{2}:=\frac{\max \left(3 / 2, a_{1}\right)}{\min \left(1 / 2,1 / 4 a_{0}^{2}, a_{0}\right)} \exp \left(c_{1} T\right) .
$$

Since the right-hand side of the above inequality does not depend on $\tau$, we can take the least upper bound of the left side with respect to $\tau$ from 0 to $T$. Thus, inequality (3.4) holds, where $c:=c_{2}^{1 / 2}$.

\section{Solvability of the Problem}

From inequality (3.1), it follows that operator $L: E \rightarrow F$ is continuous, while from inequality (3.4) it follows that the range of operator $L$ is closed in $F$ and, therefore, there is the continuous inverse operator $L^{-1}$ yielding the solution. In other words, this means that operator $L$ is a linear homeomorphism from the space $E$ on the closed set $R(L) \subset F$. To prove that problem (1.1)-(1.3) has a unique solution, it remains to show that $R(L)=F$.

Theorem 3: Let the conditions of Theorem 2 hold, and let the coefficient a $(t)$ have bounded derivatives up to the second order. Then, for any functions $f \in B_{2}^{m, 0}(Q)$ and $\varphi \in L^{2}(0, b)$, there is a unique solution $u=L^{-1} \mathcal{F}$ of problem $(1.1)-(1.3)$, where $\mathscr{F}=(f, \varphi)$, and

$$
\|u\|_{E} \leq c\left(\|f\|_{B_{2}^{m, 0}(Q)}+\|\varphi\|_{L^{2}(0, b)}\right)
$$

where constant $c$ is independent of $u$.

Proof: To prove Theorem 3, we need the following proposition.

Proposition: Let $D_{0}(L)=\{u / u \in D(L), \ell u=0\}$ and let the conditions of Theorem 3 hold. If for $v \in B_{2}^{m, 0}(Q)$ and for all $u \in D_{0}(L)$, 


$$
(\mathcal{L u , v})_{B_{2}^{m, 0}(Q)}=0
$$

then $v$ vanishes almost everywhere on $Q$.

Proof of the Proposition: Assume that relation (4.1) holds for any function $u \in D_{0}(L)$. Using this fact we can express (4.1) in a special form. First define $h$ by the formula

Let $\frac{\partial u}{\partial t}$ be a solution of

$$
h:=-\int_{t}^{T} \frac{\partial}{\partial \tau}\left(a(\tau) \frac{\partial u}{\partial \tau}\right) d \tau .
$$

and let

$$
a(t) \frac{\partial u}{\partial t}=h
$$

We, now, have

$$
D_{s}(L):=\{u / u \in D(L): u=0 \text { for } t \leq s\}
$$

$$
v=-\frac{\partial}{\partial t}\left(a(t) \frac{\partial u}{\partial t}\right)
$$

Relations (4.2) and (4.3) imply that $u$ is in $D_{0}(L)$. It possesses, in fact, a higher order of smoothness, and we have the following result:

Lemma 2: If the conditions of the proposition are met, then the function $u$ defined by (4.2) and (4.3) has derivatives with respect to $t$ up to the second order belonging to the space $B_{2}^{m, 0}\left(Q_{s}\right)$, where $Q_{s}=(0, b) \times(s, T)$.

Proof of Lemma 2: To prove Lemma 2, we will use the following $t$-averaging operators: Let $\omega \in C^{\infty}(\mathbb{R}), \omega \geq 0 ; \omega=0$ in a neighborhood of $t=0$ and $t=T$, and outside the interval $(0, T)$, and let $\int_{\mathbb{R}} \omega(t) d t=1$. We consider the operators $\rho_{\epsilon}$ defined by the formula

$$
\left(\rho_{\epsilon} w\right)(x, t)=\frac{1}{\epsilon} \int_{0}^{T} \omega\left(\left(\frac{s-t}{\epsilon}\right)\right) w(x, s) d s \text { for } w \in B_{2}^{m, 0}(Q) .
$$

The above operators have the following properties:

P1: The function $\rho_{\epsilon} w \in C^{\infty}(Q)$ and it vanishes in a neighborhood of $t=T$ if $w \in B_{2}^{m, 0}(Q)$, and $\rho_{\epsilon} u \in D_{s}(L)$ if $u \in D_{s}(L)$.

P2: If $w \in B_{2}^{m, 0}(Q)$, then $\left\|\rho_{\epsilon} w-w\right\|_{B_{2}^{m, 0}(Q)} \rightarrow 0$ when $\epsilon \rightarrow 0$, and $\left\|\rho_{\epsilon} w\right\|_{B_{2}^{m, 0}(Q)} \leq$ $\|w\|_{B_{2}^{m, 0}(Q)}{ }^{*}$

P3: $\frac{d^{k}}{d t^{k}} \rho_{\epsilon} u=\rho_{\epsilon} \frac{d^{k} u}{d t^{k}}$ for $k=1,2$ if $u \in D_{s}(L)$.

P4: If $w \in B_{2}^{m, 0}(Q)$ then,

$$
\left\|\frac{\partial}{\partial t}\left(a(t) \rho_{\epsilon} w-\rho_{\epsilon} a(t) w\right)\right\|_{B_{2}^{m, 0}(Q)} \rightarrow 0, \text { when } \epsilon \rightarrow 0 .
$$

Proofs of properties $P 1-P 4$ are similar to the proofs of the corresponding properties obtained in [3] (see Lemma 9.1).

Applying the operators $\rho_{\epsilon}$ and $\frac{\partial}{\partial t}$ to equation (4.2), we obtain

$$
a(t) \frac{\partial}{\partial t} \rho_{\epsilon} \frac{\partial u}{\partial t}=\frac{\partial}{\partial t}\left(a(t) \rho_{\epsilon} \frac{\partial u}{\partial t}-\rho_{\epsilon} a(t) \frac{\partial u}{\partial t}\right)-a^{\prime}(t) \rho_{\epsilon} \frac{\partial u}{\partial t}+\frac{\partial}{\partial t} \rho_{\epsilon} h .
$$


It follows that

$$
\begin{gathered}
\left\|a(t) \frac{\partial}{\partial t} \rho_{\epsilon} \frac{\partial u}{\partial t}\right\|{ }_{B_{2}^{m}, 0}^{2}(Q) \\
+\| c_{3}\left(\left\|\rho_{\epsilon} \frac{\partial u}{\partial t}\right\|{ }_{B_{2}^{m}, 0}^{2}(Q)\right. \\
\left.+\frac{\partial}{\partial t} \rho_{\epsilon} h\left\|_{B_{2}^{m, 0}(Q)}^{2}+\right\| \frac{\partial}{\partial t}\left(a(t) \rho_{\epsilon} \frac{\partial u}{\partial t}-\rho_{\epsilon} a(t) \frac{\partial u}{\partial t}\right) \|_{B_{2}^{m, 0}(Q)}^{2}\right)
\end{gathered}
$$

where $c_{3}=\max \left(3 c_{1}, 3\right)$.

By virtue of properties $P 1-P 4$ of the $t$-averaging operators and by inequality (2.3), we have

$$
\left(\left\|\frac{\partial^{2} u}{\partial t^{2}}\right\|_{B_{2}^{m, 0}(Q)}^{2} \leq c_{4}\left\|\frac{\partial u}{\partial t}\right\|_{L^{2}(Q)}^{2}+\left\|\frac{\partial}{\partial t} \rho_{\epsilon} h\right\|_{B_{2}^{m, 0}(Q)}^{2}\right)
$$

where $c_{4}:=\max \left(c_{3} b^{2 m} /\left(a_{0}^{2} 2^{m}\right), 1 / a_{0}^{2}\right)$. This yields the proof of Lemma 2 .

Now, we will prove the proposition. Replace $v$ in (4.1) by its representation (4.4). We have

$$
\begin{gathered}
-2 \operatorname{Re}\left(\frac{\partial u}{\partial t}, \frac{\partial}{\partial t}\left(a(t) \frac{\partial \bar{u}}{\partial t}\right)\right)_{B_{2}^{m, 0}\left(Q_{s}\right)} \\
-2 \operatorname{Re}\left((-1)^{m} a(t) \frac{\partial^{2 m}}{\partial x^{2 m}}, \frac{\partial}{\partial t}\left(a(t) \frac{\partial \bar{u}}{\partial t}\right)\right)_{B_{2}^{m, 0}\left(Q_{s}\right)}=0 .
\end{gathered}
$$

We write the remaining two terms of (4.5) in the form:

$$
\begin{gathered}
-2 \operatorname{Re}\left(\frac{\partial u}{\partial t}, \frac{\partial}{\partial t}\left(a(t) \frac{\partial \bar{u}}{\partial t}\right)\right)_{B_{2}^{m, 0}\left(Q_{s}\right)} \\
=\left\|a^{1 / 2}(s) \sigma^{m} \frac{\partial u(x, s)}{\partial t}\right\|_{L^{2}(0, b)}^{2}-\left\|a^{\prime 1 / 2}(t) \sigma^{m} \frac{\partial u}{\partial t}\right\|_{L^{2}\left(Q_{s}\right)}^{2} \\
-2 \operatorname{Re}\left((-1)^{m} a(t) \frac{\partial^{2 m} u}{\partial x^{2 m}, \frac{\partial}{\partial t}}\left(a(t) \frac{\partial \bar{u}}{\partial t}\right)\right)_{B_{2}^{m, 0}\left(Q_{s}\right)} \\
=2\left\|a(t) \frac{\partial u}{\partial t}\right\|_{L^{2}\left(Q_{s}\right)}^{2}+\operatorname{Re}\left(a^{\prime}(T) u(x, T), a(T) \bar{u}(x, T)\right)_{L^{2}(0, b)} \\
-\left\|a^{\prime}(t) u\right\|_{L^{2}\left(Q_{s}\right)}^{2}-\operatorname{Re}\left(a^{\prime \prime}(t) u, a(t) \bar{u}\right)_{L^{2}\left(Q_{s}\right)^{\circ}}
\end{gathered}
$$

Elementary calculations, starting from (4.6) and (4.7), yield the inequalities

$$
\begin{gathered}
a_{0}\left\|\frac{\partial u(x, s)}{\partial t}\right\|_{B_{2}^{m}(0, b)}^{2} \leq c_{1}\left\|\frac{\partial u}{\partial t}\right\|_{B_{2}^{m, 0}\left(Q_{s}\right)}^{2}-2 \operatorname{Re}\left(\frac{\partial u}{\partial t}, \frac{\partial}{\partial t}\left(a(t) \frac{\partial \bar{u}}{\partial t}\right)\right)_{B_{2}^{m, 0}\left(Q_{s}\right)}, \\
2 a_{0}^{2}\left\|\frac{\partial u}{\partial t}\right\|_{L^{2}\left(Q_{s}\right)}^{2}+a_{0} c_{0}\|u(x, T)\|_{L^{2}(0, b)}^{2} \\
\leq-2 \operatorname{Re}\left((-1)^{m} a(t) \frac{\partial^{2 m} u}{\partial x^{2 m}, \frac{\partial}{\partial t}}\left(a(t) \frac{\partial \bar{u}}{\partial t}\right)\right)_{B_{2}^{m, 0}\left(Q_{s}\right)}+\left(1 / 2 a_{1}^{2}+c_{1}^{2}+1 / 2 c_{5}^{2}\right)\|u\|_{L^{2}\left(Q_{s}\right)}^{2},
\end{gathered}
$$

where $c_{5}: \sup _{0 \leq t \leq T}\left|a^{\prime \prime}(t)\right|$.

Consequently, 


$$
\begin{aligned}
& \left\|\frac{\partial u}{\partial t}\right\|_{L^{2}\left(Q_{s}\right)}^{2}+\|u(x, T)\|_{L^{2}(0, b)}^{2}+\left\|\frac{\partial u(x, s)}{\partial t}\right\|_{B_{2}^{m}(0, b)}^{2} \\
& \leq c_{6}\left(\|u\|_{L^{2}\left(Q_{s}\right)}^{2}+\left\|\frac{\partial u}{\partial t}\right\|_{B_{2}^{m, 0}\left(Q_{s}\right)}^{2}\right),
\end{aligned}
$$

where $c_{6}:=\max \left(c_{1}, 1 / 2 a_{1}^{2}+c_{1}^{2}+1 / 2 c_{5}^{2}\right) / \min \left(a_{0}, 2 a_{0}^{2}, a_{0} c_{0}\right)$.

Inequality (4.8) is the basic of our proof. To use (4.8), we note that constant $c_{6}$ is independent of $s$. However, function $u$ in (4.8) depends on $s$. To avoid this difficulty we introduce a new function $\theta$ by the formula

$$
\theta(x, t):=\int_{t}^{T} \frac{\partial u}{\partial \tau} d \tau .
$$

Then, $u(x, t)=\theta(x, s)-\theta(x, t), u(x, T)=\theta(x, s)$, and we have

$$
\|u\|_{L^{2}\left(Q_{s}\right)}^{2} \leq 2\left(\|\theta(x, t)\|_{L^{2}\left(Q_{s}\right)}^{2}+(T-s)\|\theta(x, s)\|_{L^{2}(0, b)}^{2}\right) .
$$

Hence, if $s_{0}>0$ satisfies $0<2 c_{6}\left(T-s_{0}\right) \leq 1 / 2$, then (4.8) implies that

$$
\begin{gathered}
\left\|\frac{\partial u}{\partial t}\right\|_{L^{2}\left(Q_{s}\right)}^{2}+\left\|\frac{\partial u(x, s)}{\partial t}\right\|_{B_{2}^{m}(0, b)}^{2}+\|\theta(x, s)\|_{L^{2}(0, b)}^{2} \\
\leq 4 c_{6}\left(\left\|\frac{\partial u}{\partial t}\right\|_{B_{2}^{m, 0}\left(Q_{s}\right)}^{2}+\|\theta(x, t)\|_{L^{2}\left(Q_{s}\right)}^{2}\right)
\end{gathered}
$$

for all $s \in\left[T-s_{0}, T\right]$.

We denote the sum of the two terms on the right of (4.9) by $\beta(s)$. Hence, we obtain

$$
\left\|\frac{\partial u}{\partial t}\right\|_{L^{2}\left(Q_{s}\right)}^{2}-\frac{d \beta(s)}{d s} \leq 4 c_{6} \beta(s)
$$

and, consequently,

$$
-\frac{d}{d s}\left(\beta(s) \exp \left(4 c_{6} s\right)\right) \leq 0
$$

Integrating (4.10) over $(s, T)$ and taking into account that $\beta(T)=0$, we obtain

$$
\beta(s) \exp \left(4 c_{6} s\right) \leq 0
$$

It follows from (4.11), that $v=0$ almost everywhere on $Q_{T}-s_{0}$. Proceeding this way step by step along the rectangle with side $s_{0}$, we prove that $v=0$ almost everywhere on $Q$. This completes the proof of the proposition.

Now, we will prove Theorem 3. For this purpose it is sufficient to prove that the range $R(L)$ of $L$ is dense in $F$.

Suppose that, for some $V=\left(v, v_{0}\right) \in{ }^{\perp} R(L)$,

$$
(\ell u, v)_{B_{2}^{m, 0}(Q)}+\left(\ell u, v_{0}\right)_{L^{2}(0, b)}=0 .
$$

We must prove that $V=0$. Putting $u \in D_{0}(L)$ into (4.12) we obtain

$$
(\ell u, v)_{B_{2}^{m, 0}(Q)}=0, \quad u \in D(L) .
$$


Hence, the proposition implies that $v=0$. Thus, (4.12) takes the form

$$
\left(\ell u, v_{0}\right)_{L^{2}(0, b)}=0, \quad u \in D(L) .
$$

Since the range of operator $\ell$ is everywhere dense in $L^{2}(0, b)$, the above relation implies that $v_{0}=0$. Hence, $V=0$. This proves Theorem 3 .

\section{References}

[1] Benouar, N.E. and Yurchuk, Mixed problem with an integral condition for parabolic equations with an integral condition for parabolic equations with the Bessel operator, Differents. Uravn. 27:12 (1991), 2094-2098.

[2] Cannon, J.R., The solution of the heat equation subject to the specification of energy, Quart. Appl. Math. 21:2 (1963), 155-160.

[3] Gårding, L., Cauchy's Problem for Hyperbolic Equations, University of Chicago 1957.

[4] Ionkin, N.I., Solution of boundary value problems in heat conduction theory with nonlocal boundary conditions, Differents. Uravn. 13:2 (1977), 294-304.

[5] Ionkin, N.I., Stability of a problem in heat conduction theory with nonlocal boundary conditions, Differents. Uravn. 15:7 (1979), 1279-1283.

[6] Ionkin, N.I. and Moiseev, E.I., A problem for the heat conduction equation with two-point boundary condition, Differents. Uravn. 15:7 (179), 1284-1295.

[7] Kamynin, N.I., A boundary value problem in the theory of the heat conduction with nonclassical boundary condition, Th., Vychisl., Mat., Fiz. 4:6 (1964), 1006-1024.

[8] Kartynnik, A.V., Three point boundary value problem with an integral space variables conditions for second order parabolic equations, Differents. Uravn. 26 (1990), 1568-1575.

[9] Samarskii, A.A., Some problems in differential equations theory, Differents. Uravn. 16:11 (1980), 1925-1935.

[10] Yurchuk, N.I., Mixed problem with an integral condition for certain parabolic equations, Differents. Uravn. 22:12 (1986), 2117-2126. 


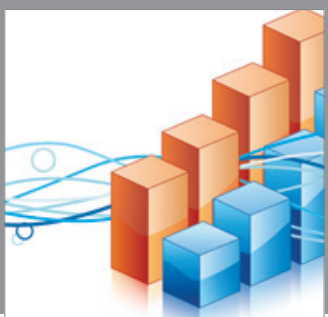

Advances in

Operations Research

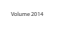

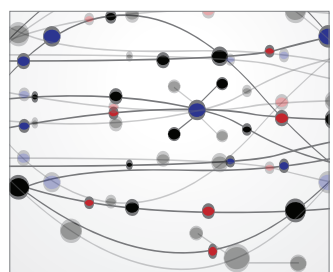

\section{The Scientific} World Journal
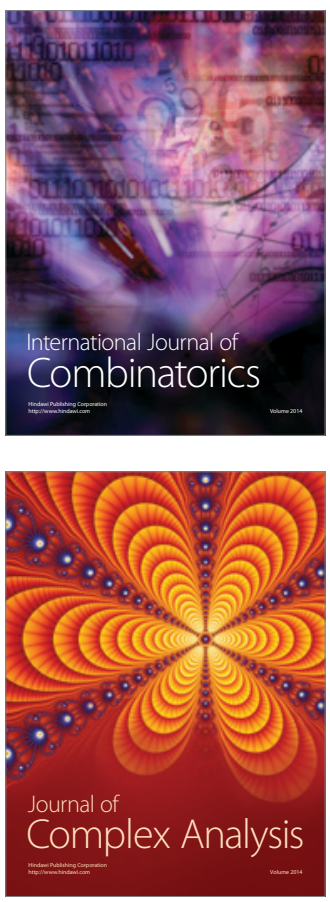

International Journal of

Mathematics and

Mathematical

Sciences
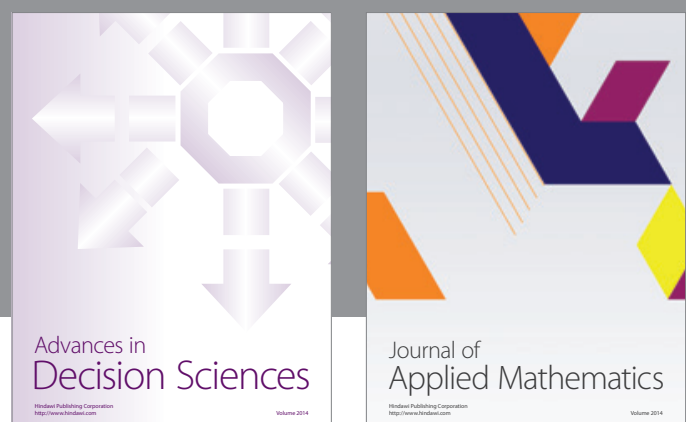

Journal of

Applied Mathematics
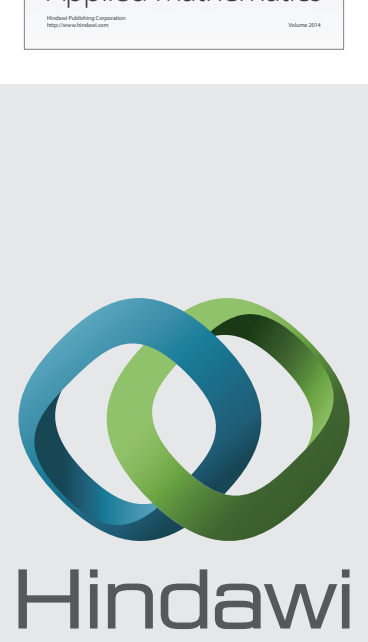

Submit your manuscripts at http://www.hindawi.com
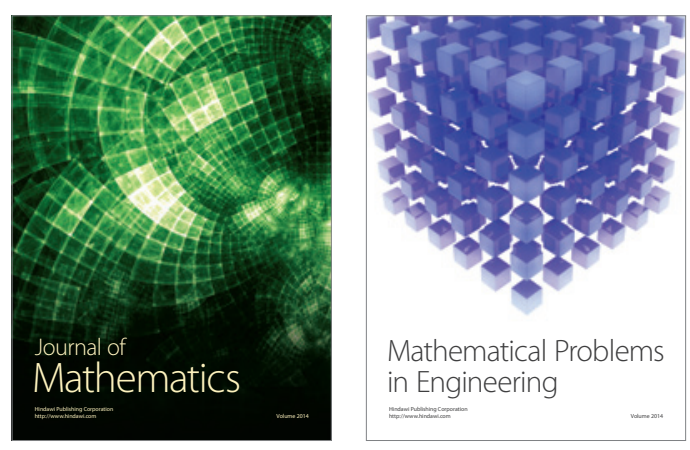

Mathematical Problems in Engineering
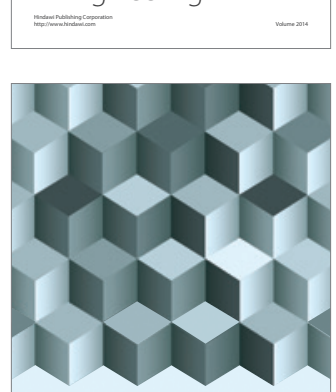

Journal of

Function Spaces
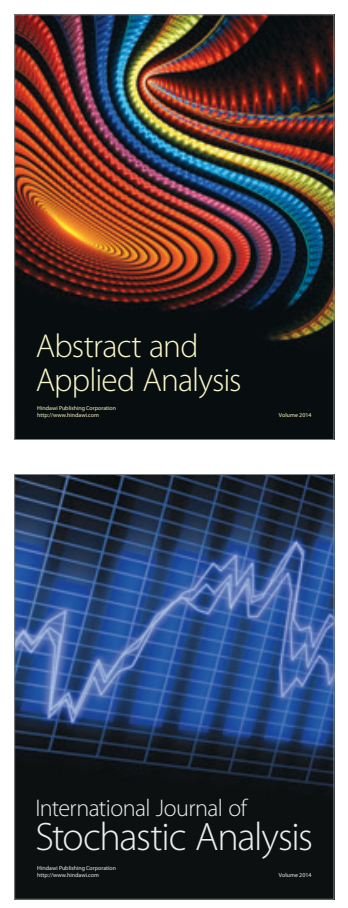

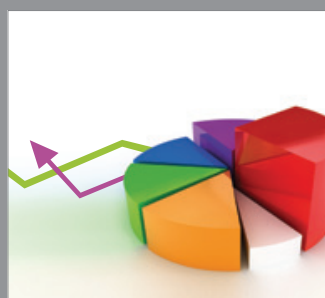

ournal of

Probability and Statistics

Promensencen
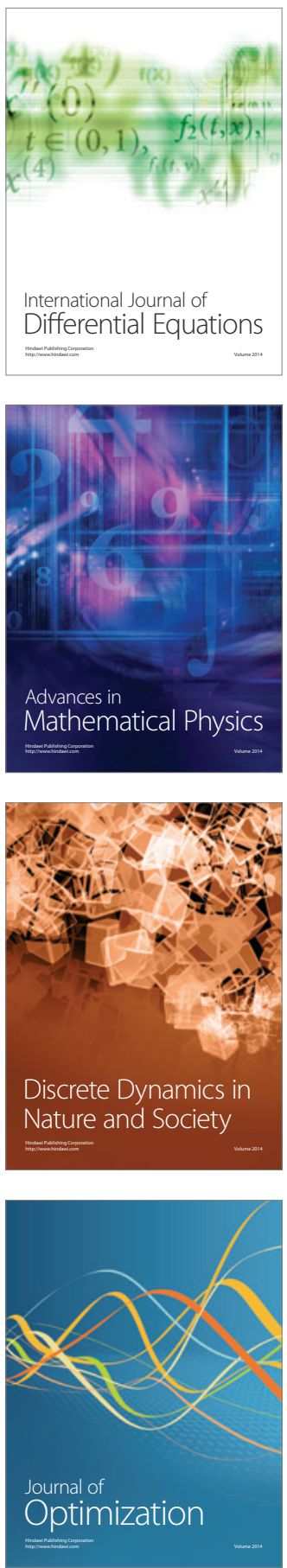